\title{
Observaciones Experimentales sobre la Fuerza Motriz en la Propagación de Grietas de Fatiga
}

\author{
Jorge A. R. Durán, Cecilia T. Hernández y José A. de Castro \\ Universidade Federal Fluminense, Escola de Engenharia Industrial Metalúrgica de Volta Redonda, \\ Av. dos Trabalhadores 420 Vila, 27255-125, Volta Redonda R.J.-Brasil \\ (e-mail: duran@vm.uff.br)
}

Recibido Mar. 09, 2011; Aceptado Abr. 27, 2011; Versión Final recibida May. 06, 2011

\section{Resumen}

Se analiza el papel que juega el factor de intensidad de las tensiones efectivo y aplicado ( $\Delta \mathrm{K}_{\text {eff }} \mathrm{y}$ $\Delta \mathrm{K}_{\mathrm{app}}$ ) como fuerzas motrices en la propagación de grietas por fatiga. Para poder utilizar confiablemente el $\Delta \mathrm{K}_{\text {eff }}$ en diseño es preciso demostrar que éste solo depende de los mismos parámetros incluidos en la expresión del $\Delta \mathrm{K}_{\text {app }}$ (la tensión nominal, el tamaño de la grieta y la función de geometría). Con el objetivo de verificar este comportamiento se hicieron ensayos de $\mathrm{K}$ creciente y decreciente en una misma probeta. El factor de cierre $\mathrm{U}=\Delta \mathrm{K}_{\text {eff }} / \Delta \mathrm{K}_{\text {app }}$ mostró una variación acentuada con el tamaño de la grieta en todos los ensayos por lo que no debe usarse el $\Delta \mathrm{K}_{\text {eff }}$ en diseño mecánico. Los datos experimentales también se muestran en tres dimensiones encontrando similares resultados, aunque no es posible generalizar la conclusión con los datos disponibles.

Palabras clave: cierre de las grietas, propagación de grietas por fatiga, factores de intensidad de las tensiones, diseño mecánico

\section{Experimental Observations on the Driving Force in Fatigue Crack Propagation}

\begin{abstract}
The role of the effective and applied stress intensity factors $\left(\Delta \mathrm{K}_{\mathrm{app}}\right.$ and $\left.\Delta \mathrm{K}_{\text {eff }}\right)$ as driving forces in fatigue crack growth is analyzed. To make a reliable use of $\Delta \mathrm{K}_{\text {eff }}$ in design it is needed to show that this parameter depends only on the same parameters that are included in the expression of $\Delta \mathrm{K}_{\mathrm{app}}$ (the nominal stress, the crack size and the geometry function). To verify this behavior experimental $\mathrm{K}$-increasing and $\mathrm{K}$-decreasing tests were done with the same specimen. The closure factor $U=\Delta K_{\text {eff }} \Delta K$ showed an accentuated variation with crack size in all the tests, so $\Delta K_{\text {eff }}$ should not be used for design purposes. The experimental data are also presented in three dimensions finding similar results, although it is not possible to generalize the results with the data available.
\end{abstract}

Keywords: crack closure, fatigue crack propagation, stress intensity factors, mechanical design 


\section{INTRODUCCIÓN}

Después de algunas dificultades iniciales en los años sesenta, como relatado por Paris et al. en 1999, quedó demostrado que el rango del factor de intensidad de las tensiones aplicado $\Delta \mathrm{K}_{\mathrm{app}}=\mathrm{K}_{\max }-\mathrm{K}_{\min }$ de la mecánica de la fractura lineal elástica MFLE es la principal fuerza motriz de la PGF. Como la fatiga es básicamente un proceso de plasticidad microcíclica, la mayor parte de la propagación ocurre bajo condiciones predominantemente elásticas y $\Delta \mathrm{K}_{\text {app }}$ indica bien la severidad de la distribución de tensiones alrededor de la grieta en crecimiento (Schijve, 2009). La relación de carga $\mathrm{R}=\sigma_{\min } / \sigma_{\max }$, donde $\sigma_{\max } \mathrm{y} \sigma_{\min }$ son las tensiones máximas y mínimas, respectivamente, ejerce influencia en la forma sigmoidal de las curvas PGF, de una manera similar al efecto de las cargas medias en las curvas tensión x número de ciclos de la fatiga de alto ciclo. De hecho el parámetro $\mathrm{R}$ es utilizado de forma casi unánime para representar las cargas medias en ciclos de amplitud constante. La figura 1, por ejemplo, muestra las curvas de propagación para valores de $\mathrm{R}$ pulsantes pero bien diferentes de un acero utilizado en tubos.

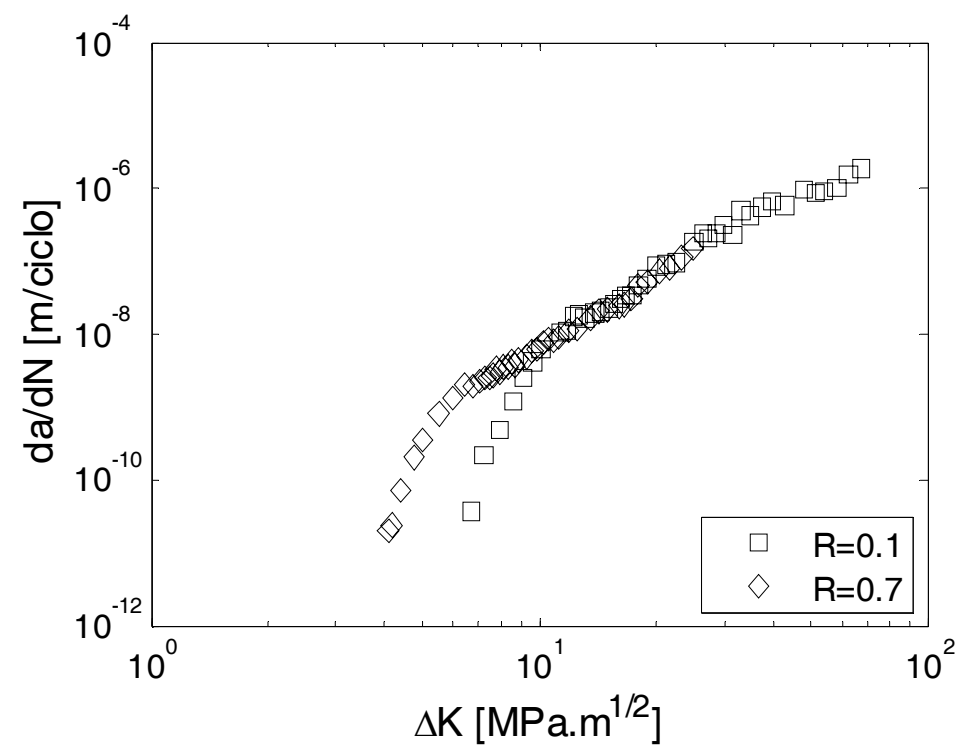

Fig. 1: Curvas de PGF del acero para tubos API5L X-60 medidas en diferentes cargas medias (Durán, 2001).

De esta manera, despreciando la dependencia con la historia de carga que puede ocurrir como resultado de la deformación plástica, las tasas de PGF pueden expresarse como una función de los parámetros $\Delta \mathrm{K}_{\mathrm{app}}$ y $\mathrm{R}$ :

$$
\frac{\mathrm{da}}{\mathrm{dN}}=\mathrm{h}\left(\Delta \mathrm{K}_{\mathrm{app}}, \mathrm{R}\right)
$$

La singularidad de las tensiones en la punta de la grieta (definida por $\Delta \mathrm{K}_{\mathrm{app}}$ ) estará presente mientras la grieta esté completamente abierta. Como apuntado por Elber en 1970, después de algún crecimiento y debido a las diferencias de tamaño entre las zonas plásticas cíclicas y monotónicas, un rastro de deformaciones plásticas positivas va quedando atrás de la grieta. Durante un ensayo de PGF, por ejemplo, el contacto físico entre los bordes de la grieta hace que la probeta parezca más rígida y su comportamiento se asemeja al que tuviera con una grieta menor. De acuerdo con este análisis, solo la parte del ciclo de carga (o de tensiones) $\Delta \sigma_{\text {eff }}=\sigma_{\max }-\sigma_{\mathrm{op}}$, donde $\sigma_{\mathrm{op}}$ es la tensión de abertura de la grieta, que corresponde a la grieta completamente abierta, puede ser tomado en cuenta para calcular el así llamado rango efectivo del factor de intensidad de las tensiones $\Delta \mathrm{K}_{\text {eff. }}$ Esta descripción corresponde al bien conocido fenómeno de cierre inducido por plasticidad que ha resultado en algunas décadas de investigación y algunos millares de artículos al respecto (Okayasu et al. 2006). 
Si la variación del $\Delta \mathrm{K}_{\text {eff }}$ con el tamaño de la grieta, como ocurre con el $\Delta \mathrm{K}_{\mathrm{app}}$, se limita al radicando y a la función normalizada $\mathrm{f}(\mathrm{a} / \mathrm{w})\left(\Delta \mathrm{K}_{\mathrm{app}}=\Delta \sigma(\pi \mathrm{a})^{1 / 2} \cdot \mathrm{f}(\mathrm{a} / \mathrm{w})\right.$, donde "a" es el tamaño de la grieta, " $\mathrm{w}$ " es el ancho de la probeta y $\mathrm{f}(\mathrm{a} / \mathrm{w})$ es una función de la geometría), lo que significa que para cargas de amplitud constante $\sigma_{\text {op }}$ no varía con el espesor, por ejemplo, como observado por Elber en sus experimentos con aluminio 2024-T3, el factor de cierre " $U$ " puede ser definido como:

$$
U=\frac{\Delta K_{\text {eff }}}{\Delta K_{\text {app }}}=\frac{\Delta \sigma_{\text {eff }} \sqrt{\pi a} f(a / w)}{\Delta \sigma \sqrt{\pi a} f(a / w)}=\frac{\Delta \sigma_{\text {eff }}}{\Delta \sigma}
$$

Definido de esta forma, "U" representa el porciento de $\Delta \mathrm{K}_{\text {app }}$ que la grieta efectivamente experimenta en su punta y $\Delta \mathrm{K}_{\text {eff }}$ describe el campo de tensiones en la punta de la grieta cuando ésta está completamente abierta. Cuanto mayor "U", menor el cierre, luego éste debería ser correctamente llamado de factor de abertura.

Si el cierre inducido por plasticidad es el responsable por los efectos de "R" en las curvas de PGF, como cree una buena parte de los expertos, esto significa que ambas variables, $\Delta K_{a p p}$ y $R$, de la ecuación (1) pueden remplazarse por un único parámetro $\Delta \mathrm{K}_{\text {eff }}$ a través de una función $U(R) \cdot \Delta K$. Por esta razón, muchos esfuerzos se han hecho con el objetivo de obtener correlaciones empíricas $\mathrm{U}(\mathrm{R})$ para diseño mecánico. Por ejemplo, para todo el rango teórico de valores de $\mathrm{R}$ $(-1<R<1)$ la siguiente relación fue propuesta por Schijve en 1981:

$$
U=0.55+0.33 R+0.12 R^{2}
$$

Para soldaduras de acero inoxidable y $0 \leq R \leq 0.5$, Singh et al (2007) han propuesto $U=0.66-2.41 \cdot R+7 \cdot R^{2}$. En cualquier caso la introducción de $U(R)$ permite que la ecuación (1) sea rescrita como:

$$
\frac{\mathrm{da}}{\mathrm{dN}}=\mathrm{q}\left(\Delta \mathrm{K}_{\text {eff }}\right)=\mathrm{q}(\mathrm{U}(\mathrm{R}) \times \Delta \mathrm{K})
$$

El éxito de la ecuación 4 puede medirse si los datos para diferentes relaciones " $R$ " son

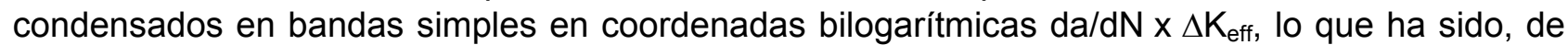
hecho, observado (Donald y Paris 1999). Recientemente, sin embargo, Kujawski (2003) ha demostrado que la condensación de datos experimentales para varios $R$ 's en una única línea no se puede considerar como una confirmación de la solidez del parámetro $\Delta \mathrm{K}_{\text {eff. }}$.

Si el principio de la similitud es rescrito en la forma de Elber, estos resultados significan que ensayos hechos en algún "R" son suficientes para encontrar (empíricamente) la función "q" de la ecuación (4) para un dado material y utilizarla para el diseño con cualquier combinación $\Delta \mathrm{K} \times \mathrm{R}$, lo que sin dudas es de enorme importancia desde el punto de vista del ingeniero de diseño estructural.

La relación $\sigma_{\mathrm{op}} / \sigma_{\max }$, que es también utilizada como una medida cuantitativa del cierre inducido por plasticidad, puede ser fácilmente deducida a partir de la expresión $\Delta \sigma_{\text {eff }}=\sigma_{\max }-\sigma_{\mathrm{op}}$ (ya mencionada encima) y de la ecuación (2). Utilizando la propuesta de Schijve para "U" (ecuación (3)) resulta que:

$$
\frac{\sigma_{o p}}{\sigma_{\max }}=1-U(1-R)=0.45+0.22 R+0.21 R^{2}+0.12 R^{3}
$$

Otra relación $\left(\sigma_{\mathrm{op}}=\alpha \cdot \sigma_{\max }\left[1-\left(\sigma_{\max } / \mathrm{S}_{\mathrm{yc}}\right)^{2}\right]+\beta \cdot \sigma_{\min }\right)$ ha sido propuesta por DuQuesnay (1991). En este caso $\alpha$ e $\beta$ son parámetros ajustables ( $\alpha=0.45$ y $\beta=0.2$ para aleaciones de aluminio) y $S_{y c}$ es una constante próxima del límite de fluencia cíclico del material. Las propuestas de DuQuesnay 
y Schijve se plotan junto con la línea de no cierre $\sigma_{o p}=\sigma_{\min }$ en la figura 2. Para un dado valor de $\sigma_{\max }$ el incremento en los valores de "R" significa que ambos, $\sigma_{\mathrm{m}} \mathrm{y} \sigma_{\min }$, donde $\sigma_{\mathrm{m}}$ es la tensión promedio, también se incrementan, lo que tiende a mantener la grieta abierta $\mathrm{y}$, consecuentemente, menos cierre debe esperarse. Este análisis lógico es consistente con el comportamiento de $\sigma_{\mathrm{op}} / \sigma_{\max }$ observado en la figura 2 . Debe recordarse, sin embargo, que se parte de la consideración de que $\sigma_{\mathrm{op}}$ no varía con el espesor o la sección remanente.

Conocimientos básicos de la mecánica de los materiales, sin embargo, permiten argumentar que el cierre debe, de alguna forma, estar relacionado con el estado de tensiones en la punta de la grieta (tensión plana $\sigma_{p}$ o deformación plana $\varepsilon_{p}$ ). De hecho Newman (1981), utilizando la técnica de elementos finitos y a partir de una modificación del modelo de la tira de fluencia de Dugdale (1960), mostró que otros parámetros como la tensión máxima y el espesor $\left(\sigma_{p} \circ \varepsilon_{p}\right)$, además de $R$, tienen una influencia importante en el cierre de las grietas. Los cálculos de vida residual que no lleven en cuenta estos parámetros producen resultados no conservativos (Newman Jr. y Ruschau 2007, Meggiolaro y Castro 2003). Es importante destacar, sin embargo, que la guía de ensayos de PGF (norma ASTM E647-99) no define el espesor de las probetas, lo que implícitamente significa que considera el $\Delta \mathrm{K}_{\mathrm{app}} \mathrm{y}$ no el $\Delta \mathrm{K}_{\text {eff }}$ como la fuerza motriz de la propagación.

Como se observa, el tema del cierre de las grietas todavía despierta mucho interés en la comunidad científica. No siendo posible resumir la dependencia de da/dN con el $\Delta \mathrm{K}_{\text {eff, }}$ porque éste varía con otros parámetros que no se incluyen en la ecuación 4, entonces, una opción sería hacer ensayos variando estos parámetros, midiendo el cierre y decidiendo si el da/dN es mejor relacionado con el $\Delta \mathrm{K}_{\text {eff }} \mathrm{O}$ con el $\Delta \mathrm{K}_{\text {app. }}$. Este es el objetivo principal del presente trabajo.

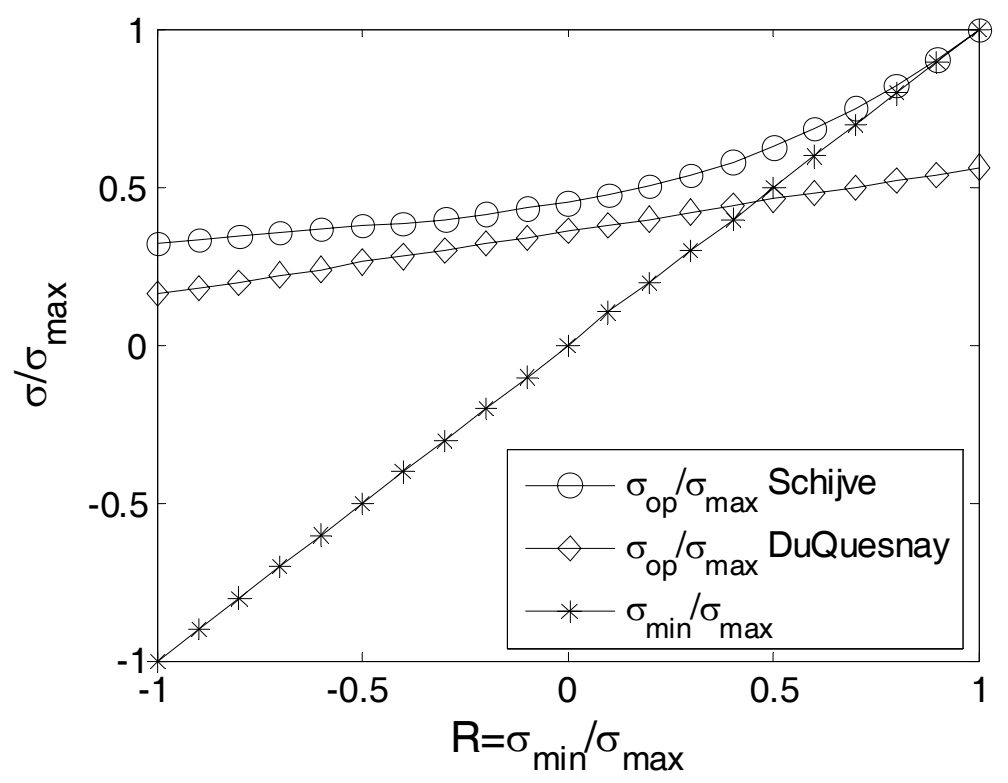

Fig. 2: Curvas de cierre $\sigma_{\mathrm{op}} / \sigma_{\max }$ de acuerdo con DuQuesnay (1991) (para aluminio y asumiendo $\sigma_{\max } / \mathrm{S}_{\mathrm{yc}}=0.45$ ) y Schijve (1981), y de no cierre $\sigma_{\min } / \sigma_{\max }=R$.

\section{METODOLOGÍA EXPERIMENTAL Y RESULTADOS}

Los experimentos fueron realizados en dos tipos de materiales y utilizando dos tipos de probetas, como resumido en la tabla 1 . En el caso de probetas de acero 4340, dos espesores fueron usados. Para cada serie de datos, la tabla 1 también muestra un número de identificación (Id.) que será usado más adelante en el trabajo. Los ensayos de PGF fueron realizados bajo control de amplitud de carga (constante) en un sistema servohidráulico de $100 \mathrm{KN}$ de capacidad. Las cargas fueron siempre pulsantes con bajas relaciones " $R$ ", como mostrado en la tabla 1. De acuerdo con la figura 2 se espera un cierre considerable en estas relaciones. 
Como dicho antes, ensayos de $\mathrm{K}$ creciente y $\mathrm{K}$ decreciente fueron realizados en la misma probeta hasta donde su ancho lo permitiera. Para evitar los efectos de historia, un cuidadoso procedimiento de disminución de la carga aplicada fue llevado a cabo en los ensayos de $\mathrm{K}$ decreciente, lo que permitió obtener gradientes normalizados de $\mathrm{K}$ alrededor de $-0,082 \mathrm{~mm}^{-1}$.

Tabla 1: Identificación y series de datos para las probetas utilizadas en este estudio.

\begin{tabular}{|c|c|c|c|c|c|}
\hline Id. & Material & Tipo de Probeta & Espesor B $(\mathrm{mm})$ & Ancho w $(\mathrm{mm})$ & $\mathrm{R}$ \\
\hline 1 & Acero 1020 & Compacto de Tracción C(T) & 10 & 50 & 0.1 \\
\hline 2 & Acero 4340 & En forma de Disco DC(T) & 3.2 & 55 & 0.05 \\
\hline 3 & Acero 4340 & En forma de Disco DC(T) & 24 & 55 & 0.05 \\
\hline
\end{tabular}

La curva de tamaño de la grieta versus el número de ciclos (axN) para los diferentes ensayos se muestra en la figura 3. Algunos cambios abruptos en la inclinación de las curvas, indicando períodos de aceleración y desaceleración de la grieta se notan fácilmente, lo que está de acuerdo con los ya mencionados ensayos de $\mathrm{K}$ creciente y decreciente. Estas variaciones en las tasas de PGF fueron siempre medidas de acuerdo con la norma ASTM E647-99 y, para el ensayo Id1 y a lo largo de la probeta, se muestran en la figura 4.

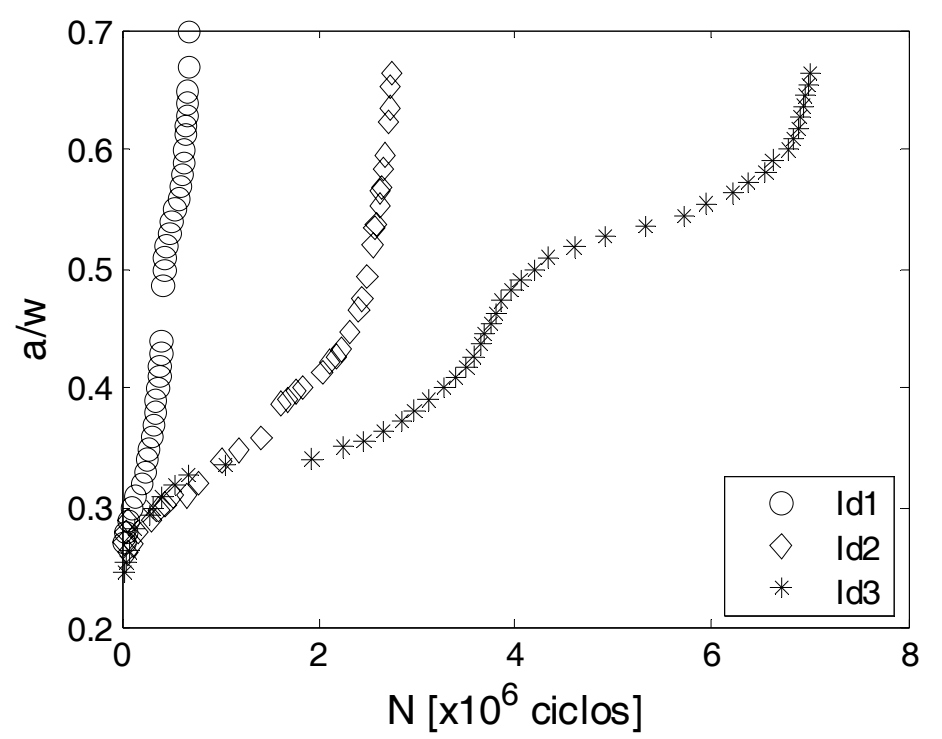

Fig. 3: Curvas de tamaño de la grieta versus número de ciclos (axN) para los diferentes ensayos. La serie de datos para cada identificador está de acuerdo con la tabla 1.

En todos los casos un extensómetro de resistencia eléctrica permitió la adquisición de los datos de deformación de la parte trasera DPT de la probeta, los cuales fueron utilizados con dos objetivos: medición del cierre a diferentes tamaños de la grieta (junto con los datos de carga) y medición del tamaño de la grieta. Para cumplir el último objetivo fue utilizada una calibración experimental propuesta por Gilbert et al. en 1994. En comparación con otros métodos (correlación de imagen digital, por ejemplo), las medidas de DPT se han mostrado más eficaces para medir cargas de cierre (Matos y Nowell 2009). 


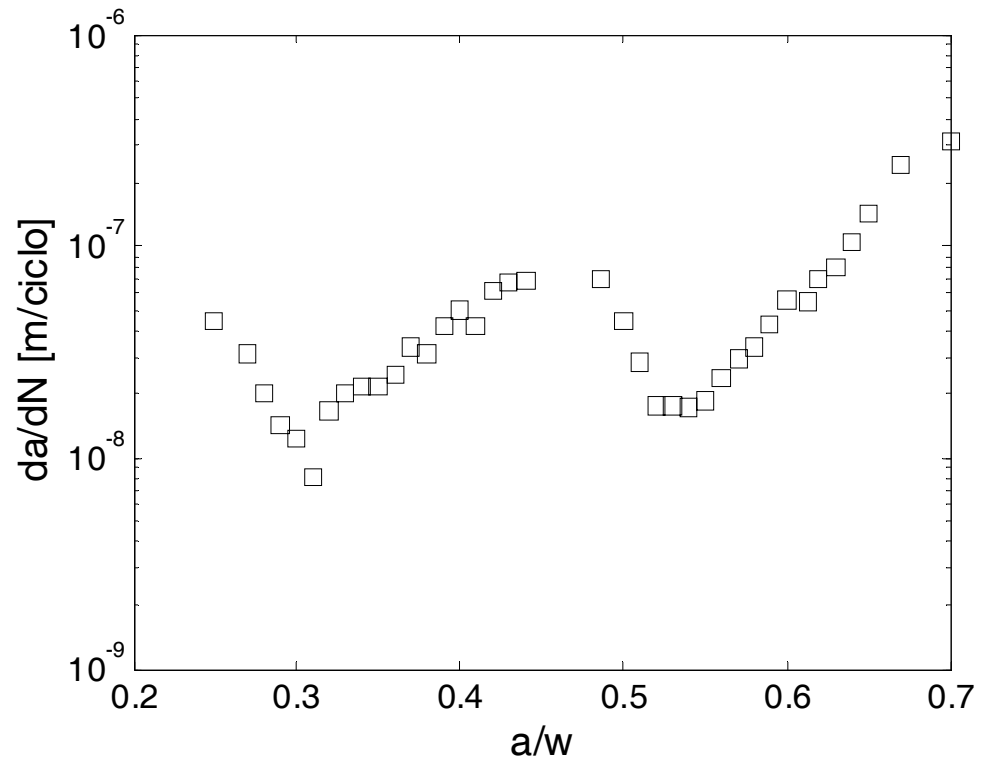

Fig. 4: Las tasas de PGF varían de acuerdo con el régimen de K. En este caso, dos ensayos de K creciente $\mathrm{y}$ dos de $\mathrm{K}$ decreciente fueron posibles a lo largo de la probeta.

Las medidas de cierre fueron hechas después de parar el ensayo en diferentes tamaños de grieta, aplicar una rampa lineal de carga (a una tasa de $0,04 \mathrm{KN} / \mathrm{s}$ ) y descarga en el rango de $\Delta P=P_{\max }-P_{\min }$ que estaba siendo utilizado en el momento de la parada de la grieta. Alrededor de seis mil puntos de deformación versus carga fueron adquiridos en cada tamaño de grieta. La figura 5 presenta una pequeña muestra de estos datos, específicamente para la probeta Id.3 (ver tabla 1) en el régimen de $\mathrm{K}$ creciente y a un tamaño de grieta normalizado $a / w=0,4$. Es interesante notar que los caminos de carga y descarga son prácticamente los mismos y que la pendiente constante del gráfico se observa aproximadamente encima de los $8 \mathrm{KN}$.

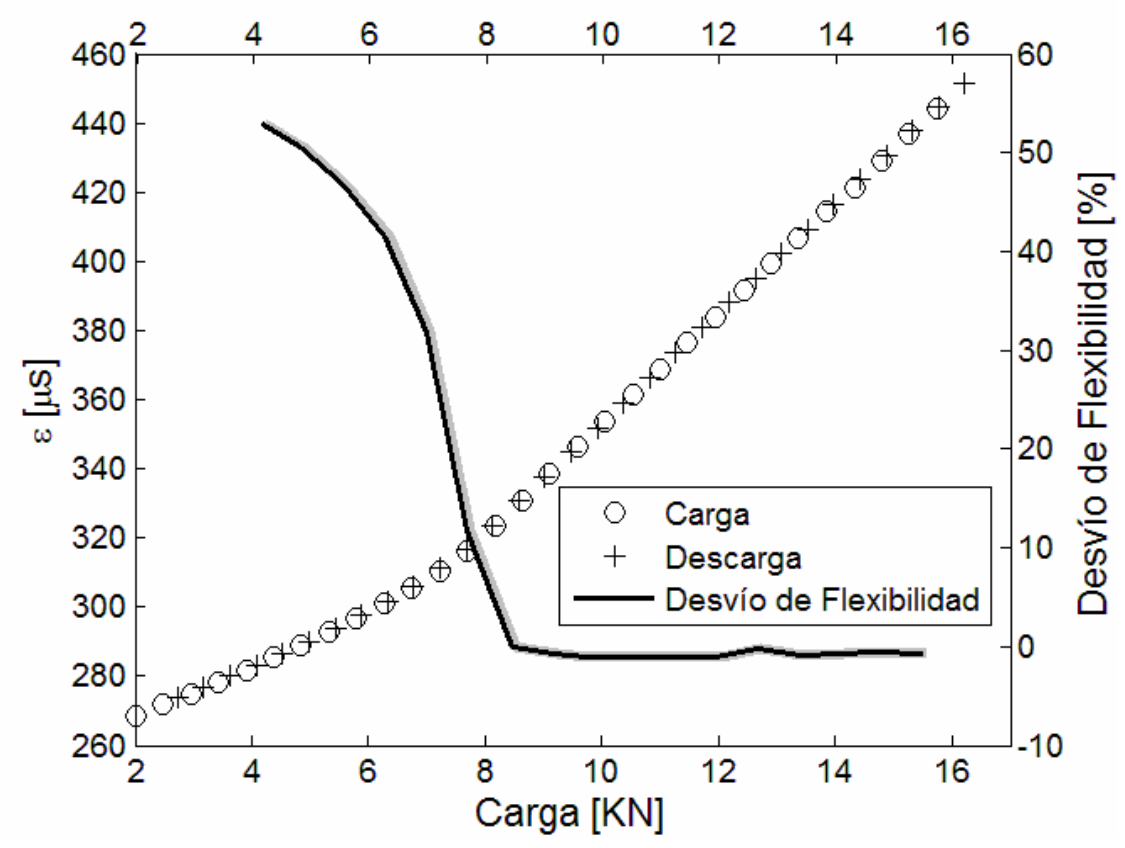

Fig. 5: Gráfico de dos ejes mostrando 1) los datos de carga y descarga versus deformación trasera $\varepsilon[\mu \mathrm{s}$ ] y 2) la curva carga versus desvío de la flexibilidad después del procesamiento de los datos utilizando un código computacional especialmente desarrollado para este fin $\left(P_{o p}=8,3 K N\right)$.

Un código computacional fue especialmente desarrollado para procesar estos datos y determinar la carga de abertura de acuerdo con el método del $0,2 \%$ de desvío de la flexibilidad (ASTM E64799). Los resultados se muestran en la figura 5 e indican una carga de abertura $P_{o p}=8,3 \mathrm{KN}$ para 
aquella serie de datos. Teniendo los rangos aplicado y efectivo $\left(\Delta \mathrm{K}_{\text {eff, }} \Delta \mathrm{K}_{\mathrm{app}}\right)$ de los factores de intensidad de las tensiones, es posible visualizar la variación del factor de cierre "U", según la ecuación 2, con el tamaño normalizado de la grieta "a/w", lo que se hace en la figura 6.

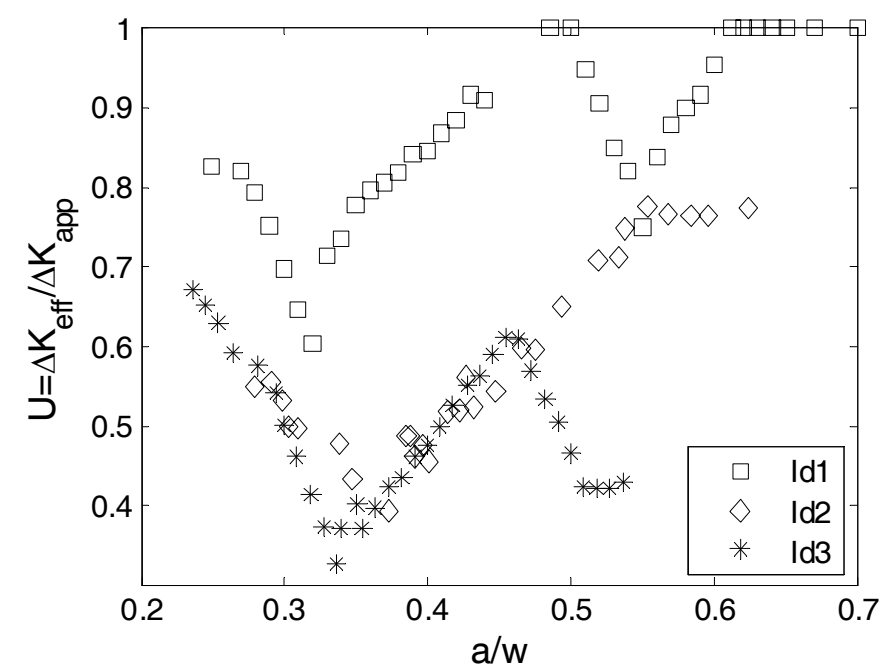

Fig. 6: Comportamiento del factor de cierre versus el tamaño normalizado de la grieta a/w para los diferentes ensayos. Las series de datos correspondientes a cada identificador están especificadas en la tabla 1.

\section{DISCUSIÓN}

La expresión de "K" para las probetas compactas de tracción preferencialmente aparece en función de la carga aplicada "P":

$K=\frac{P}{B \sqrt{w}} f\left(\frac{a}{w}\right)$

De esta forma la dependencia con el tamaño de la grieta se limita a la función normalizada f(a/w). Los experimentos realizados en este trabajo fueron hechos forzando una variación lineal del $\Delta \mathrm{K}_{\mathrm{app}}(\mathrm{a} / \mathrm{w})$, sea por la disminución gradual de la carga aplicada ( $\mathrm{K}$ decreciente), sea por la aplicación de una amplitud de carga constante ( $K$ creciente). Las tendencias de $U(a / w)$ mostradas en la figura 6 , a pesar de lineales, claramente indican que ni el $\mathrm{K}_{\mathrm{op}} \mathrm{y}$ consecuentemente, ni el $\Delta \mathrm{K}_{\text {eff }}$ siguen la variación $\Delta \mathrm{K}_{\mathrm{app}}(\mathrm{a} / \mathrm{w})$. En un sentido más matemático, la variación observada del $\mathrm{U}(\mathrm{a} / \mathrm{w})$ significa que:

$\frac{\mathrm{K}_{\mathrm{op}}}{\mathrm{K}_{\max }}=\frac{\mathrm{P}_{\mathrm{op}}}{\mathrm{P}_{\max }} \mathrm{g}\left(\frac{\mathrm{a}}{\mathrm{w}}\right)$

lo que indica que, de acuerdo con las cargas de abertura medidas en este trabajo, existe una variación cuantitativa del factor de intensidad de las tensiones $\mathrm{K}_{\mathrm{op}}$ a lo largo del tamaño de la grieta, y que esta variación no es llevada en cuenta por el factor de geometría f(a/w). Como un ejemplo de esto, la figura 7 muestra el comportamiento de los factores de intensidad medidos y aplicados a lo largo de la grieta para el ensayo Id3. Variaciones como esta ya han sido reportadas en el pasado (Vecchio et al. 1986). 


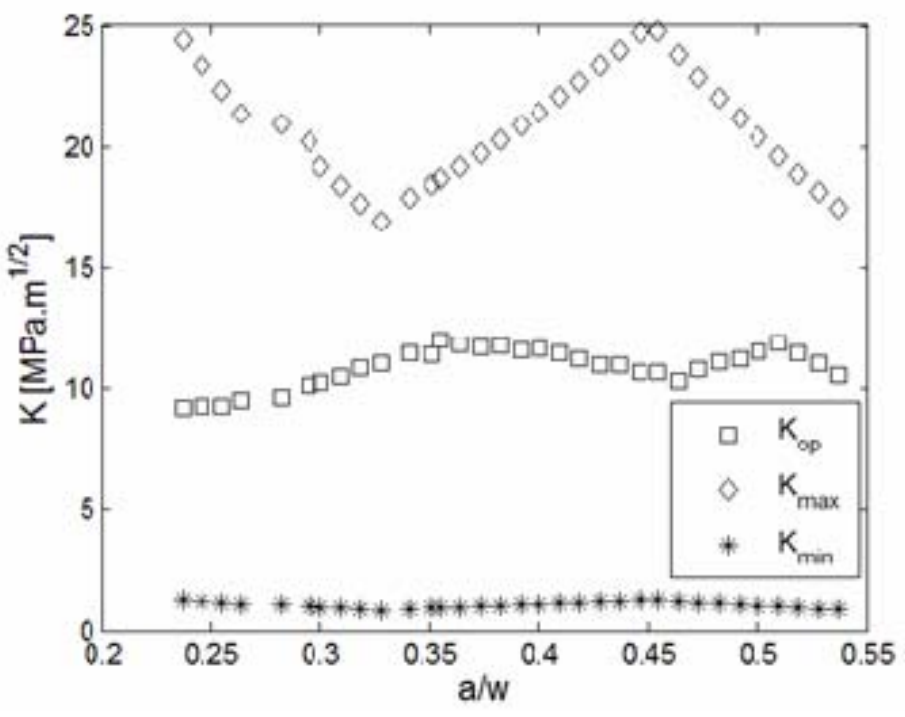

Fig. 7: Comportamiento de los factores de intensidad de las tensiones máximo y mínimo ( $\mathrm{K}_{\max }$ and $\left.\mathrm{K}_{\min }\right)$ y del factor de abertura $\left(\mathrm{K}_{\mathrm{op}}\right)$ medido durante el ensayo Id.3. Como el $\mathrm{K}_{\mathrm{op}}$ fue calculado por la ecuación (6) es evidente que existe una variación, aquí llamada de $\mathrm{g}(\mathrm{a} / \mathrm{w})$, que no es considerada por el factor de geometría $\mathrm{f}(\mathrm{a} / \mathrm{w})$.

Como afirmado anteriormente, este resultado es atribuido al efecto de la variación en la sección resistente residual de las probetas y también, en parte, a la conocida dificultad en obtener medidas consistentes de cierre de grietas. El método del $0,2 \%$ de desvío de la flexibilidad, utilizado en este trabajo, ha sido considerado muy conservativo, sobre todo en la región del umbral $\Delta \mathrm{K}_{\mathrm{th}}$, por Donald y Paris (1999) así como por otros que estos autores mencionan en su trabajo, además de sensible a la posición en que se miden las deformaciones (Lugo et al., 2011). Una buena revisión de las técnicas disponibles para medición y análisis de las cargas de abertura puede ser encontrada en Stoychev y Kujawski (2003). La evaluación de otros métodos de determinación de las cargas de abertura, sin embargo, está fuera del alcance de este trabajo.

Volviendo a la discusión sobre la variación $\mathrm{K}_{\mathrm{op}}(\mathrm{a})$, esto no significa que el principio de la similitud, en el sentido de Elber (el mismo $\Delta a$ en ciclos de $\Delta \mathrm{K}_{\text {eff }}$ similares) no podría ser aplicado. En el caso de que las tasas de PGF da/dN permanezcan aproximadamente constantes en una amplia faja de tamaños de grieta para los mismos valores efectivos de $\Delta \mathrm{K}_{\text {eff }}$, entonces el cierre inducido por plasticidad gobernaría el diseño mecánico bajo estas circunstancias. Por otra parte, la permanencia del $\Delta \mathrm{K}_{\mathrm{app}}$ constante implicaría en una confirmación de la validez de la filosofía tradicional de diseño. Estos análisis aparecen a continuación.

Los datos adquiridos en este trabajo permiten la construcción de la tabla 2 a partir de las siguientes consideraciones. Para cada ensayo fueron seleccionadas el promedio de algunas tasas de PGF con, como máximo $15 \%$ de coeficiente de variación, y las correspondientes fuerzas motrices, $\Delta \mathrm{K}_{\mathrm{app}}$ y $\Delta \mathrm{K}_{\text {eff }}$ para diferentes relaciones a/w. En figuras similares a la (4) esto significa trazar líneas horizontales que interceptan las curvas de los puntos experimentales en más de una ocasión y seleccionar los respectivos factores $\Delta \mathrm{K}$. Después se calculan el coeficiente de variación y el promedio de los datos da/dN y $\Delta \mathrm{K}$. Los datos de la tabla 2 también se plotan en la figura 8. Para cada valor promedio de tasa de PGF, independientemente del ensayo y por tanto del material y del espesor de la probeta, los valores medidos del $\Delta \mathrm{K}_{\text {eff }}$ tienen un coeficiente de variación mayor de que el coeficiente del $\Delta \mathrm{K}_{\text {app }}$.

Finalmente, los datos para los ensayos Id.2 e Id.3 se plotan en coordenadas $\Delta \mathrm{K}, \mathrm{K}_{\max } \mathrm{y}$ da/dN, como sugerido por el enfoque unificado (Sadananda y Vasudevan 2004), en la figura 9. De 
acuerdo con este método la PGF obedece a dos fuerzas motrices con sus respectivos umbrales, $\mathrm{K}_{\max } \mathrm{y} \Delta \mathrm{K}$. El parámetro $\mathrm{R}$ no se considera una fuerza motriz porque no presenta umbral de propagación. Las curvas de propagación constituyen planos para $\mathrm{R}=$ constante en coordenadas $\left(\Delta \mathrm{K}, \mathrm{K}_{\max }, \mathrm{da} / \mathrm{dN}\right)$.

Tabla 2: Coeficiente de variación CV de los datos de $\Delta \mathrm{K}_{\text {app }}$ y $\Delta \mathrm{K}_{\text {eff }}$ adquiridos a diferentes relaciones a/w para aproximadamente las mismas tasas da/dN (menos de $15 \%$ de CV) de PGF.

\begin{tabular}{|c|c|c|c|}
\hline & & \multicolumn{2}{|c|}{ CV [\%] } \\
\hline \multirow{4}{*}{ Id3 } & $\begin{array}{c}\text { da/dN } \\
{[\mathrm{m} / \text { priclo }]}\end{array}$ & $\Delta \mathrm{K}$ & $\Delta \mathrm{K}_{\text {eff }}$ \\
\hline \multirow{3}{*}{ Id2 } & $6.1 \mathrm{E}-09$ & 8.17 & 12.54 \\
\cline { 2 - 4 } & $3.4 \mathrm{E}-09$ & 7.89 & 16.05 \\
\cline { 2 - 4 } & $1.5 \mathrm{E}-09$ & 6.19 & 11.39 \\
\cline { 2 - 4 } & $5.9 \mathrm{E}-09$ & 3.78 & 9.05 \\
\hline \multirow{3}{*}{ Id1 } & $3.9 \mathrm{E}-09$ & 4.61 & 12.43 \\
\cline { 2 - 4 } & $2.6 \mathrm{E}-09$ & 5.27 & 9.34 \\
\cline { 2 - 4 } & $6.6 \mathrm{E}-08$ & 4.84 & 7.65 \\
\hline & $3.8 \mathrm{E}-08$ & 7.33 & 10.62 \\
\hline
\end{tabular}

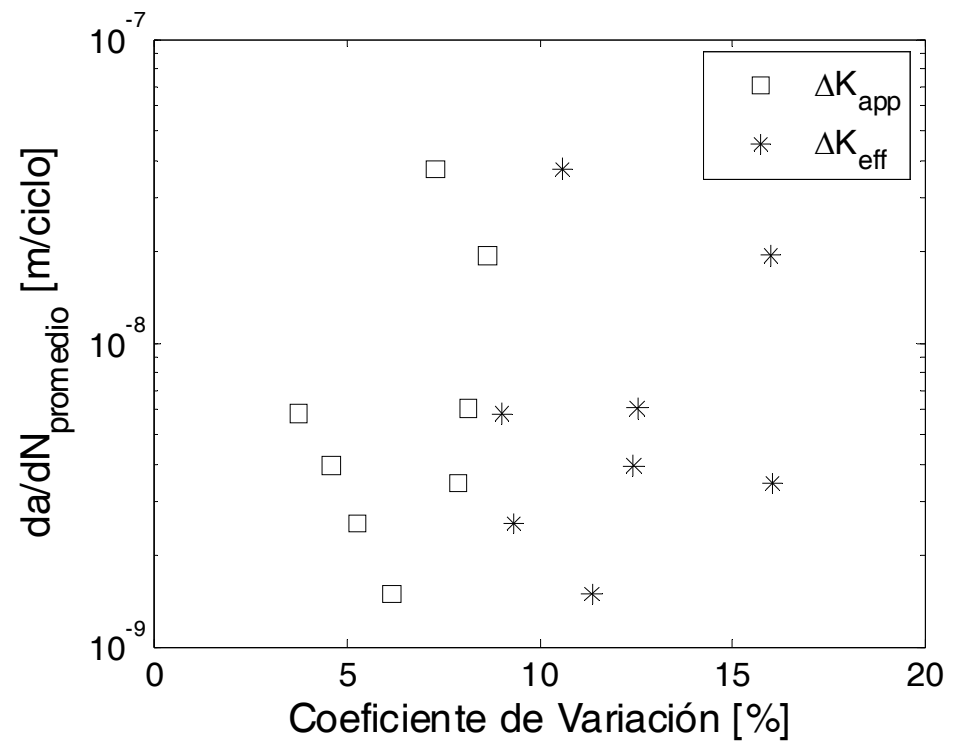

Fig. 8: Representación gráfica de los datos de la tabla 2. Para cada valor promedio de las tasas $\mathrm{da} / \mathrm{dN}$, independientemente del ensayo y por tanto del material y del espesor, los datos de $\Delta \mathrm{K}_{\text {eff }}$ presentan un mayor coeficiente de variación que los datos de $\Delta \mathrm{K}_{\mathrm{app}}$.

Cada uno de estos planos forma un ángulo de $\theta=\tan ^{-1}(1-R)$ con el eje $K_{\max }$. Como sucede en el caso del $\Delta \mathrm{K}_{\mathrm{app}}$, los puntos $\Delta \mathrm{K}_{\text {eff }} \mathrm{KK}_{\max } \mathrm{xda} / \mathrm{dN}$ deberían también mostrar un buen ajuste con los planos de $R=$ constante (recordando que $K_{\max }=\Delta K /(1-R)$ ), desde que $\Delta K_{\text {eff }}$ represente una fuerza motriz confiable del proceso de PGF. Sin embargo, los datos en la figura 9 no parecen alinearse bien con ningún plano en este espacio. De acuerdo con estos resultados no es posible considerar el cierre como una variable de proyecto contra la PGF. 

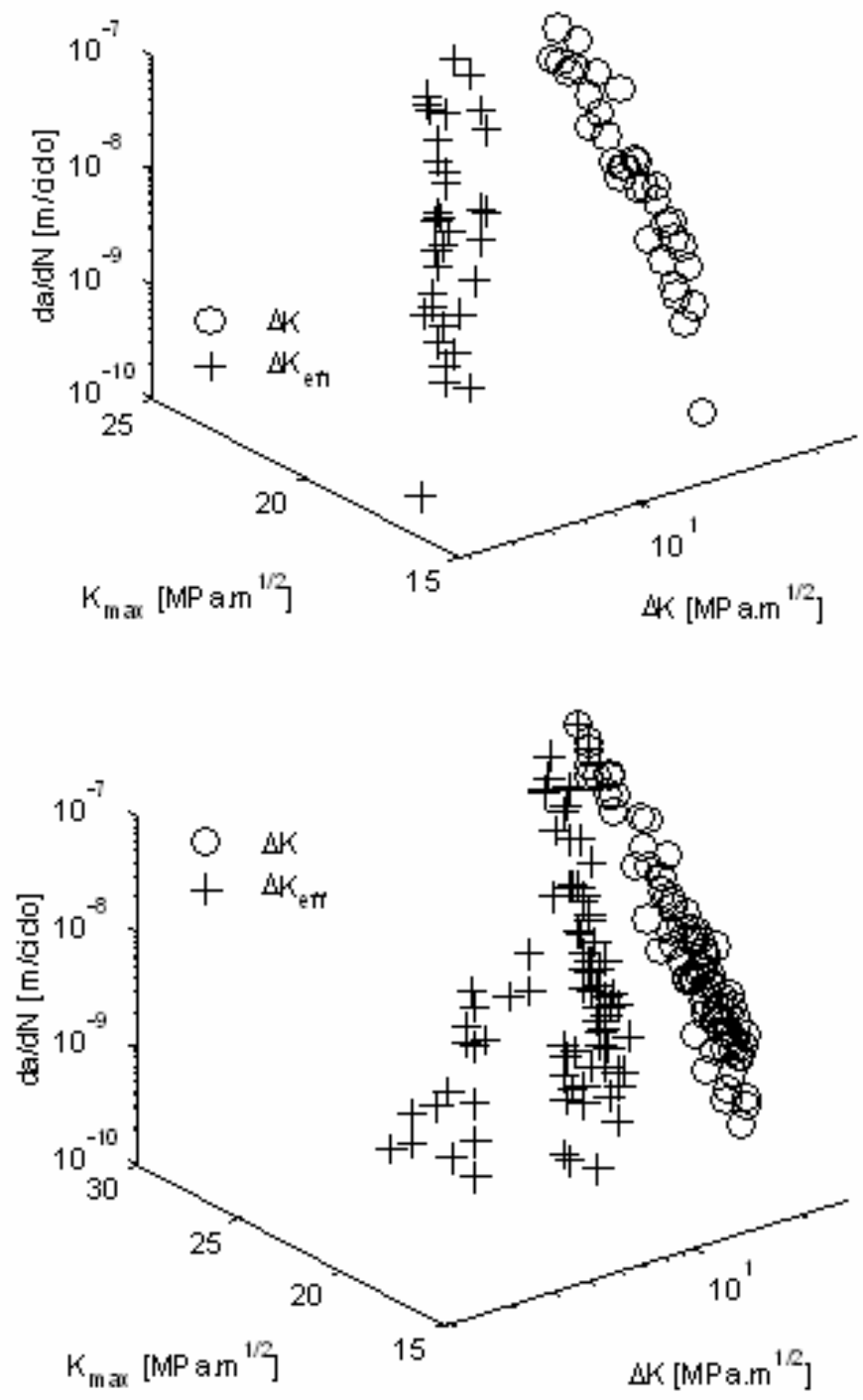

Fig. 9: Datos de propagación de grietas por fatiga obtenidos en el presente trabajo para los ensayos Id. 2 e Id. 3 con acero 4340, probeta en forma de disco, ancho de $55 \mathrm{~mm}$ y espesores 3.2 $\mathrm{mm}$ para id2 y de $24 \mathrm{~mm}$ para id3,

\section{CONCLUSIONES}

De acuerdo con los resultados experimentales obtenidos en este trabajo se llega a las siguientes conclusiones:

1 - El diseño contra la PGF incluyendo los efectos de $R$ (que se supone que sean debido al mecanismo de cierre inducido por plasticidad) solo puede ser llevado a cabo después de la introducción de alguna función empírica $g(a / w)$ en el factor de cierre $U(R, g(a / w)) \cdot \Delta K=\Delta K_{\text {eff. }}$ Desde luego, esto es imposible desde el punto de vista práctico, en lo que constituye una dificultad enorme del diseño mecánico contra la propagación basada en el cierre de las grietas.

2 - Siendo el coeficiente de variación una medida de la incertidumbre en el valor de una variable y considerando que, de acuerdo con los resultados de este trabajo el $C V$ del $\Delta \mathrm{K}_{\text {eff }}>C V$ del $\Delta \mathrm{K}_{\mathrm{app}}$ para tasas da/dN aproximadamente iguales en diferentes $\mathrm{a} / \mathrm{w}$, puede afirmarse que la filosofía tradicional de diseño basada en curvas da/dNx $\Delta \mathrm{K}_{\text {app }}$ continua siendo la mejor opción para abordar el problema de la propagación de grietas por fatiga. 
3 - Los datos de PGF en función de $\Delta K_{a p p}$ y $K_{\text {máx }}$ satisfacen el método de los dos parámetros mientras que en función de $\Delta \mathrm{K}_{\text {eff }} \mathrm{y} \mathrm{K}_{\max }$ no. A pesar de ser un fuerte indicio de que, para este caso particular, tanto el cierre de las grietas como el $\Delta \mathrm{K}_{\text {eff }}$ no deben ser considerados como parámetros de diseño, no es posible generalizar esta conclusión y una investigación más profunda se impone.

\section{REFERENCIAS}

ASTM E647-99, Standard Test Method for Measurement of Fatigue Crack Growth Rates, Annual Book of ASTM Standards, vol. 11.03, (1999).

Matos PFP de, Nowell D, Experimental and numerical investigation of thickness effects in plasticity-induced fatigue crack closure, International Journal of Fatigue 31, 1795-1804, (2009).

Donald K., P.C. Paris, An evaluation of $\Delta$ Keff estimation procedures on 6061-T6 and 2024-T3 aluminum alloys, International Journal of Fatigue, 21, S47-S57, (1999).

Dugdale DS, Yielding of Steel Sheets containing Slits, Journal of the Mechanics and Physics of Solids, 8, 100-104, 1960.

DuQuesnay DL. Fatigue damage accumulation in metals subjected to high mean stress and overload cycles. PhD thesis, University of Waterloo, Waterloo, ON, Canada; (1991).

Durán, J.A.R., Modelos de Acúmulo de Dano por Plasticidade Cíclica para Previsão de Taxas de Propagação de Trincas por Fadiga, Tesis de Titulación, Dpto. de Ing. Metalúrgica, Universidade Federal do Rio de Janeiro, (2001).

Elber, W., Fatigue Crack Closure under Cyclic Tension, Engineering Fracture Mechanics: 2, 37-45, (1970).

Gilbert, C.J., J.M. McNaney, R.H. Dauskardt y R.O. Ritchie, Back-Face Strain Compliance and Electrical-Potential Crack Length Calibrations for the Disk-Shaped Compact-Tension DC(T) Specimen, Journal of Testing and Evaluation, JTEVA, 22(2) pp. 117-120, (1994).

Kujawski, D., $\Delta K_{\text {eff }}$ parameter under re-examination, International Journal of Fatigue, 25, 793-800, (2003).

Lugo M., J.C. Daniewicz, Newman Jr, A mechanics based study of crack closure measurement techniques under constant amplitude loading, International Journal of Fatigue 33, 186-193, (2011).

Meggiolaro MA, Castro JTP, On the dominant role of crack closure on fatigue crack growth modeling, International Journal of Fatigue, 25, 843-854, (2003).

Okayasu M, Chen D, Wang Z, Experimental study of the effect of loading condition on fracture surface contact features and crack closure behavior in a carbon steel, Engineering Fracture Mechanics 73, 1117-1132, (2006).

Newman Jr JC. A crack closure model for predicting fatigue crack growth under aircraft spectrum loading, ASTM STP 748: 53-84, (1981).

Newman Jr. JC, Ruschau JJ, The stress-level effect on fatigue-crack growth under constantamplitude loading, International Journal of Fatigue 29, 1608-1615, (2007).

Paris, P.C., H. Tada y J.K. Donald, Service load fatigue damage - a historical perspective, International Journal of Fatigue: 21, S35 - S46, (1999).

Sadananda K., A.K. Vasudevan, Crack tip driving forces and crack growth representation under fatigue, International Journal of Fatigue 26, 39-47, (2004). 
Schijve, J., Fatigue of Structures and Materials, 2a edición, Springer, The Netherlands, (2009).

Schijve, J., Some formulas for the crack opening stress level, Engineering Fracture Mechanics, 14, 461-465, (1981).

Singh PJ, Mukhopadhyay CK, Jayakumar T, Mannan SL, y Baldev Raj, Understanding fatigue crack propagation in AISI $316(N)$ weld using Elber's crack closure concept: Experimental results from GCMOD and acoustic emission techniques, International Journal of Fatigue 29, 2170-2179, (2007).

Stoychev S, D. Kujawski, Methods for crack opening load and crack tip shielding determination: a review, Fatigue and Fracture of Engineering Materials and Structures, 26, 1053-1067, (2003).

Vecchio R.S., J.S. Crompton, R.W. Hertzberg, Anomalous aspects of crack closure, International Journal of Fracture 31, R29-R33, (1986). 